Ann. Génét. Sél. anim., I972, 4 (3), 445-449.

\title{
DIFFÉRENCES ENTRE SOUCHES OU ENTRE CROISEMENTS POUR LE DIMORPHISME SEXUEL DU POIDS A PLUSIEURS AGES CHEZ LA POULE $\left({ }^{1}\right)$
}

\author{
H. AYOUB et P. MÉRAT \\ Laboratoire de Génétique factorielle, \\ Centre national de Recherches zootechniques, I. N. R. A., \\ 78 - Jouy-en-Josas \\ RÉSUMÉ
}

Des interactions souche $\times$ sexe hautement significatives ont été observées, pour le poids à 8 semaines ou à ro mois d'âge. Elles correspondent à des variations d'amplitude non négligeable du rapport du poids moyen dans les deux sexes. Elles peuvent être d'origine génétique, les conditions d'élevage des différentes souches étant rendues aussi comparables que possible.

On observe, d'autre part, des différences légères mais significatives entre des croisements et les populations parentales pour le dimorphisme sexuel du poids à 6 et à $\mathrm{I} 2$ semaines, mais elles ne manifestent pas la présence d'une hétérosis liée au chromosome sexuel (" homogametic heterosis ").

\section{INTRODUCTION}

Nous avons résumé dans un article séparé (Ayoub et MÉRAT, I97I) les recherches antérieures visant à mettre en évidence des variations génétiques du dimorphisme sexuel du poids à un âge spécifié chez les oiseaux. Dans cet article, nous avons présenté nos propres résultats concernant l'existence de différences entre familles à ce point de vue. Nous examinerons ici des différences entre souches ou entre croisements.

\section{MATÉRIEL ET MÉTHODES}

Deux groupes de données étaient à notre disposition : comparaison de souches " pures " entre elles la même année, et d'autre part de souches parentes et de leurs croisements de première génération.

(1) Ce travail représente une partie d'une thèse de Docteur-Ingénieur soutenue par H. AyouB à Paris en I970 (voir aussi Ayoub et MÉrat, I972. Ann. Génét. Sél. anim., 4, 45-59). 
Io Pour le premier type de comparaison, le matériel étudié comprend, en ce qui concerne les poids à 8 semaines, trois populations élevées en 1963 à Jouy-en-Josas, et pour le poids adulte (Io mois d'âge), 5 souches de type " ponte " élevées au domaine du Magneraud en I968. Pour chaque caractère, une analyse de variance était faite, comportant en particulier le test d'interaction souche $\times$ sexe.

$2^{\circ}$ Pour les comparaisons de souches et de leurs croisements, nous avons pu disposer d'une population dérivée de Leghorn (L 22), d'une autre dérivée en partie d'une souche Rhode-Island (M 55) et de leurs croisements réciproques, élevés ensemble en I 968 à la Station de Recherches avicoles à Nouzilly. 60 coqs (30 " $\mathrm{L} 22$ ", 30 " M 55 ") étaient utilisés comme reproducteurs, chacun avec 3 poules de chaque souche. Les mâles étaient interchangés dans une $2^{\mathrm{e}}$ période pour que chaque femelle soit accouplée à un mâle de chaque lignée. On obtenait ainsi des descendants des deux souches parentes et des croisements à l'intérieur de la même case pedigree. Sur ces données, nous présentons l'écart de chaque croisement à la moyenne des souches parentales par sexe. Une analyse de variance avec les facteurs contrôlés "sexe " et " groupe génétique " permet ensuite de tester en particulier l'interaction sexe $\times$ groupe.

Les populations du Magneraud (I er groupe de comparaisons) comportaient une proportion de $\sigma^{\star} \sigma^{*}$ gardés jusqu'à l'âge adulte de l'ordre de $30 \mathrm{p}$. I00, assez voisine dans les diverses souches comparées. Par contre, aucun tri n'était fait avant l'enregistrement des poids à 8,6 et 12 semaines respectivement dans les autres cas.

Tous les animaux étaient élevés au sol pour les périodes considérées et dans des conditions comparables à l'intérieur de chaque comparaison, les deux sexes étant mélangés dans les mêmes locaux jusqu'à Io semaines d'âge ( $\mathrm{I}^{\mathrm{er}}$ type de comparaisons) ou $\mathrm{I} 2$ semaines ( $2^{\mathrm{e}}$ type). Toutefois, pour les données de Jouy et du Magneraud, chaque souche était placée dans un local distinct.

\section{RÉSULTATS}

\section{I. - Interaction souche $\times$ sexe}

Le tableau I indique, dans les trois souches de Jouy pour le poids à 8 semaines et dans celles du Magneraud pour le poids adulte, les effectifs, valeurs moyennes par sexe et leur rapport.

TABLEAU I

Effectifs, moyennes par sexe et leur rapport pour le poids à 8 semaines et adulte dans diverses souches

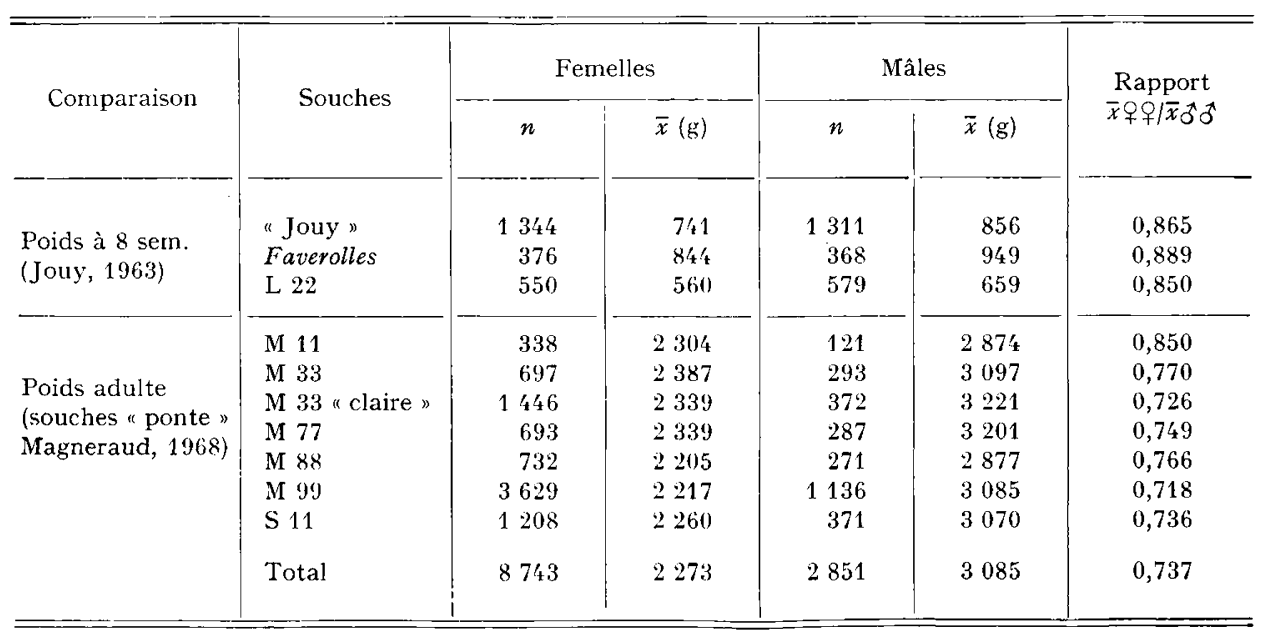


Le tableau 2 contient pour chaque comparaison le rapport de la variance interaction souche $\times$ sexe à la variance résiduelle.

On constate que l'interaction est significative dans chaque cas.

\section{TABLEAU 2}

Signification de l'interaction souches $\times$ sexe

\begin{tabular}{|c|c|c|c|c|}
\hline Comparaison & $\begin{array}{l}\text { Caractère } \\
\text { analysé }\end{array}$ & $\begin{array}{l}\text { Source de } \\
\text { variation }\end{array}$ & $\begin{array}{l}\text { Degrés de } \\
\text { liberté }\end{array}$ & $\mathrm{F}$ \\
\hline $\begin{array}{l}3 \text { souches de } \\
\text { Jouy-en-Josas }\end{array}$ & $\begin{array}{c}\text { Poids à } 8 \text { se- } \\
\text { maines }\end{array}$ & $\begin{array}{l}\text { Interaction } \\
\text { souches } \times \text { sexe } \\
\text { Résiduelle }\end{array}$ & $\begin{array}{c}2 \\
4520\end{array}$ & $2,88^{*}$ \\
\hline $\begin{array}{l}\text { Magneraud } \\
\text { (7 souches } \\
\text { "ponte") }\end{array}$ & Poids adulte & $\begin{array}{l}\text { Interaction } \\
\text { souches } \times \text { sexe } \\
\text { Résiduelle }\end{array}$ & $\begin{array}{c}6 \\
11580\end{array}$ & $34,21 * *$ \\
\hline
\end{tabular}

* Significatif au seuil 5 p. 100.

** Significatif au seuil 1 p. 100.

\section{2. - Souches parentales et leurs croisements}

Le tableau 3 présente les effectifs, valeurs moyennes par sexe et groupe et écarts des croisements à la moyenne des populations parentales, par sexe.

\section{TABLEAU 3}

Dimorphisme sexuel à 6 et 12 semaines pour des souches pures et leurs croisements

(Station de Recherches avicoles, Nouzilly)

\begin{tabular}{|c|c|c|c|c|c|c|c|c|}
\hline \multirow[b]{2}{*}{ Age } & \multirow[b]{2}{*}{$\begin{array}{l}\text { Souche ou } \\
\text { croisement }\end{array}$} & \multicolumn{3}{|c|}{ 우앙 } & \multicolumn{3}{|c|}{ పेరి } & \multirow[b]{2}{*}{$\begin{array}{l}\text { Rapport } \\
\bar{x}+q \bar{x} ठ ð\end{array}$} \\
\hline & & $n$ & $\bar{x}(g)$ & $\begin{array}{l}\text { Écart des } \\
\text { croisements } \\
\text { à la demi- } \\
\text { somme des } \\
\text { parents (g) }\end{array}$ & $n$ & $\bar{x}(\mathrm{~g})$ & $\begin{array}{l}\text { Écart des } \\
\text { croisements } \\
\text { à la demi- } \\
\text { somme des } \\
\text { parents (g) }\end{array}$ & \\
\hline 6 semaines & 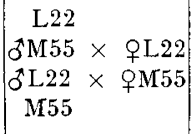 & $\begin{array}{l}442 \\
538 \\
394 \\
335\end{array}$ & $\begin{array}{l}354,6 \\
433,9 \\
402,9 \\
457,0\end{array}$ & $\begin{array}{l}+6,9 \\
-\quad 0,3\end{array}$ & $\begin{array}{l}476 \\
569 \\
375 \\
335\end{array}$ & $\begin{array}{l}402,6 \\
492,2 \\
464,7 \\
510,1\end{array}$ & $\begin{array}{l}+7,8 \\
+1,9\end{array}$ & $\begin{array}{l}0,880 \\
0,881 \\
0,867 \\
0,895\end{array}$ \\
\hline 12 semaines & 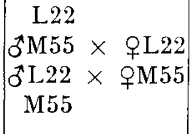 & $\begin{array}{l}559 \\
611 \\
470 \\
449\end{array}$ & $\begin{array}{ll} & 963,9 \\
1 & 127,2 \\
1 & 082,2 \\
1 & 184,4\end{array}$ & $\begin{array}{l}+\quad 4,9 \\
+\quad 0,1\end{array}$ & $\begin{array}{l}539 \\
633 \\
458 \\
451\end{array}$ & $\begin{array}{ll}1 & 202,0 \\
1 & 429,8 \\
1 & 392,1 \\
1 & 525,0\end{array}$ & $\begin{array}{r}+\quad 4,9 \\
+\quad 2,1\end{array}$ & $\begin{array}{l}0,801 \\
0,788 \\
0,777 \\
0,776\end{array}$ \\
\hline
\end{tabular}


Enfin, l'analyse de variance, avec les facteurs contrôlés " sexe " et " groupe génétique ", figure au tableau 4.

\section{TABLEAU 4}

Analyse de variance du poids à 6 et à 12 semaines par sexe et par groupe

(Souches pures et leurs croisements. Station de Recherches avicoles)

\begin{tabular}{|c|c|c|c|c|}
\hline \multirow{2}{*}{ Source de variation } & \multicolumn{2}{|c|}{ Poids à 6 semaines } & \multicolumn{2}{|c|}{ Poids à 12 semaines } \\
\hline & $\begin{array}{l}\text { degrés de } \\
\text { liberté }\end{array}$ & $\mathrm{F}$ & $\begin{array}{l}\text { degrés de } \\
\text { liberté }\end{array}$ & $\mathrm{F}$ \\
\hline Entre sexes & 1 & $442,41 * *$ & 1 & $3784,39 * *$ \\
\hline Entre groupes & 3 & $203,55^{* *}$ & 3 & $579,16 * *$ \\
\hline $\begin{array}{c}\text { Interaction } \\
\text { groupes } \times \text { sexe }\end{array}$ & 3 & 2,41 & 3 & $19,67 * *$ \\
\hline Résiduelle & 3456 & - & 4162 & 一 \\
\hline
\end{tabular}

* Significatif au seuil 1 p. 100.

** Significatif au seuil 5 p. 100.

Outre le fait que la souche " $M 55$ " est plus lourde que la " $L, 22$ » et que le croisement dont la mère est " $L, 22$ » est supérieur au croisement réciproque, dans les deux sexes et aux deux âges, l'analyse de variance suggère, à 6 semaines, une différence entre groupes pour le rapport du poids moyen des 우우 à celui des $\hat{\sigma} \widehat{\sigma}$ (rapport de la variance interaction sexe $\times$ groupe à la variance résiduelle s'approchant du seuil 5 p. roo de signification). A I2 semaines, la variance interaction est hautement significative.

\section{DISCUSSION ET CONCLUSIONS}

Des interactions souche $\times$ sexe hautement significatives apparaissent, tant pour le poids à 8 semaines (souches de Jouy) que pour le poids adulte (Magneraud). Elles correspondent à des variations d'amplitude non négligeables du rapport du poids moyen des deux sexes. Il se peut que ces interactions représentent des différences génétiques entre populations : quoique chaque souche ait été placée dans un local distinct, les conditions d'élevage de toutes les souches dans chaque comparaison étaient rendues aussi voisines que possible. D'autre part, nous avons indiqué plus haut qu'en ce qui concerne les poids adultes, le pourcentage d'élimination des coqs après 8 semaines n'était pas très différent dans les souches comparées (valeurs extrêmes 60 et 74 p. roo), et ne présente pas de corrélation apparente avec le rapport 
du poids moyen des deux sexes, de sorte que cela ne peut être une cause appréciable de différence dans ce rapport. Une question d'échelle peut aussi être écartée : il n'y a pas de relation visible, dans ces résultats, entre moyenne des deux sexes réunis et dimorphisme.

Dans l'ensemble, ces résultats peuvent se rapprocher des travaux de HoRToN et Mc BRIDE (I964).

On observe, d'autre part, des différences légères mais significatives entre croisements et populations parentales pour le dimorphisme sexuel, mais elles ne manifestent pas la présence d'une hétérosis liée au chromosome sexuel (" homogametic heterosis ») suggéré par STONAKER (I962, I963). Il est vrai que nos données portent sur des populations non ou peu consanguines, qui avaient moins de chances a priori d'extérioriser un tel phénomène. Il en est de même d'autres croisements entre souches, soit légères, soit lourdes, faits au domaine du Magneraud (CochEz, communications personnelles).

Reçu pour publication en avril 1979.

\section{REMERCIEMENTS}

Nous remercions M, J.-P. Boyer, Station de Recherches avicoles, Nouzilly, et le Dr L.-P. Cochez, Station expérimentale d'Aviculture du Magneraud, d'avoir mis à notre dispositions les données contenues dans cet article.

\section{SUMMARY}

\section{DIFFERENCES BETWEEN STRAINS OR CROSSES FOR SEX DIMORPHISM OF WEIGHT AT SEVERAL AGES IN THE FOWL.}

Highly significant sex $\times$ strain interactions were observed, for 8 week and ro months weight. They correspond to appreciable variations of the ratio of mean weight of the two sexes. They may be of genetic origin, the environment of the various strains being standardized as far as possible.

On the other hand, slight but significant differences were observed between crosses and parental strains for sex dimorphism of 6 and 12 week weight, but they do not suggest the existence of "homogametic heterosis".

\section{RÉFÉRENCES BIBLIOGRAPHIQUES}

Ayoub H., Mérat P., I972. Différences entre familles pour le dimorphisme sexuel du poids à un âge donné chez la poule. Ann. Génét. Sél. anim. 4, 45-59.

Horton J. F., Mc Bride G., r964. Genetic factors affecting sex dimorphism in body weight of meat chickens. Proc. Austr. Poult. Sci. conv., r964, 79-8I.

Stonaker H. H., ig62. Homogametic heterosis. Am. Soc. Anim. prod. 13, 23 I-238.

Stonaker H. H., I963. A genetic hypothesis for sex-mating system interaction in growth of cattle and poultry. J. anim. Sci. 22, 320-325.

Annales de Génétique animale. - 1972. 